\title{
Lp-PLA 2 activity is associated with increased risk of diabetic retinopathy: a longitudinal disease progression study
}

\author{
Moneeza K. Siddiqui ${ }^{1} \cdot$ Gwen Kennedy $^{2} \cdot$ Fiona Carr $^{1}$ • Alexander S. F. Doney ${ }^{2}$ - Ewan R. Pearson ${ }^{1} \cdot$ Andrew D. Morris $^{3}$ • \\ Toby Johnson ${ }^{4} \cdot$ Megan M. McLaughlin ${ }^{5} \cdot$ Rachel E. Williams $^{6} \cdot$ Colin N. A. Palmer $^{1}$
}

Received: 4 September 2017 / Accepted: 28 February 2018 / Published online: 6 April 2018

(C) The Author(s) 2018

\begin{abstract}
Aims/hypothesis The aim of the study was to examine the association between lipoprotein-associated phospholipase $\mathrm{A}_{2}$ $\left(\mathrm{Lp}-\mathrm{PLA}_{2}\right)$ activity levels and incident diabetic retinopathy and change in retinopathy grade.

Methods This was a cohort study of diabetic participants with serum collected at baseline and routinely collected diabetic retinal screening data. Participants with type 2 diabetes from the GoDARTS (Genetics of Diabetes Audit and Research in Tayside Scotland) cohort were used. This cohort is composed of individuals of white Scottish ancestry from the Tayside region of Scotland. Survival analysis accounting for informative censoring by modelling death as a competing risk was performed for the development of incident diabetic retinopathy from a disease-free state in a 3 year follow-up period $(n=1364)$ by stratified Lp$\mathrm{PLA}_{2}$ activity levels (in quartiles). The same analysis was performed for transitions to more severe grades.

Results The hazard of developing incident diabetic retinopathy was 2.08 times higher $(95 \%$ CI 1.64, 2.63) for the highest quartile of Lp-PLA $A_{2}$ activity compared with the lowest. Higher Lp-PLA $A_{2}$ activity levels were associated with a significantly increased risk for transitions to all grades. The hazards of developing observable (or more severe) and referable (or more severe) retinopathy

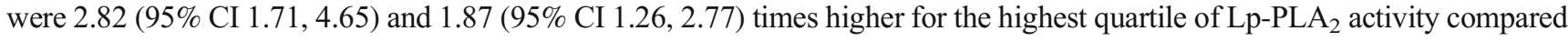
with the lowest, respectively.

Conclusions/interpretation Higher Lp-PLA 2 levels are associated with increased risk of death and the development of incident diabetic retinopathy, as well as transitions to more severe grades of diabetic retinopathy. These associations are independent of calculated LDL-cholesterol and other traditional risk factors. Further, this biomarker study shows that the association is temporally sensitive to the proximity of the event to measurement of Lp-PLA 2.
\end{abstract}

Keywords Diabetic complications $\cdot$ Electronic medical records $\cdot$ Epidemiology $\cdot$ Lipids/lipoproteins $\cdot$ Microvascular disease . Retinopathy

Electronic supplementary material The online version of this article (https://doi.org/10.1007/s00125-018-4601-7) contains peer-reviewed but unedited supplementary material, which is available to authorised users.

Colin N. A. Palmer

c.n.a.palmer@dundee.ac.uk

1 Pat McPherson Centre for Pharmacogenetics and Pharmacogenomics, University of Dundee School of Medicine, Ninewells Hospital and Medical School, Dundee DD1 9SY, UK

2 Ninewells Hospital and Medical School, University of Dundee, Dundee, UK

3 Usher Institute of Population Health Sciences and Informatics, University of Edinburgh, Edinburgh, UK

4 GlaxoSmithKline Medicines Research Centre, Gunnels Wood Road, Stevenage, UK

5 Alternative Discovery and Development, Research \& Development, GlaxoSmithKline, King of Prussia, PA, USA

6 Worldwide Epidemiology, GlaxoSmithKline, Collegeville, PA, USA 


\section{Research in context}

\section{What is already known about this subject?}

- Diabetic retinopathy is a leading cause of vision loss

- Lipoprotein-associated phospholipase $A_{2}\left(L p-P L A_{2}\right)$ is a proinflammatory enzyme that has previously been associated with cardiovascular health and diabetic macular oedema

What is the key question?

- Can Lp-PLA 2 be used to predict the incidence and progression of diabetic retinopathy?

What are the new findings?

- $\quad \mathrm{Lp}-\mathrm{PLA} \mathrm{A}_{2}$ can be used to predict both the incidence of diabetic retinopathy and its progression, in a time-sensitive manner

How might this impact on clinical practice in the foreseeable future?

- Lp-PLA 2 , a therapeutic target, can now be added to a panel of clinical parameters to determine those at highest risk, in order to provide the necessary personalised medical interventions to prevent diabetic retinopathy

$\begin{array}{ll}\text { Abbreviations } \\ \text { DARTS } & \text { Diabetes Audit and Research in Tayside Scotland } \\ \text { DBP } & \text { Diastolic blood pressure } \\ \text { DME } & \text { Diabetic macular oedema } \\ \text { DR(0-4) } & \text { Diabetic retinopathy (grade) } \\ \text { GoDARTS } & \text { Genetics of Diabetes Audit and Research in } \\ & \begin{array}{l}\text { Tayside Scotland } \\ \text { LDLc }\end{array} \\ \text { LDL-PLA } 2 & \text { Lipoprotein-associated phospholipase } \mathrm{A}_{2} \\ \text { SBP } & \text { Systolic blood pressure }\end{array}$

\section{Introduction}

Diabetic retinopathy is a leading cause of vision loss and blindness in the working age population (20-74 years of age) of most developed countries [1]. It was found to occur in $35 \%$ of people with diabetes based on a meta-analysis of multiple studies [2]. The increasing number of individuals with diabetes worldwide suggests that diabetic retinopathy is likely to be a growing contributor to vision loss and associated functional impairment in the future [3].

Risk factors associated with diabetic retinopathy include age, race/ethnicity, longer duration of diabetes, insulin dependence, younger age of diabetes onset, higher $\mathrm{HbA}_{1 \mathrm{c}}$, insulin treatment and higher blood pressure [4-10]. Studies have also shown that hyperlipidaemia, hyperglycaemia and hypertension contribute to the pathogenesis of diabetic retinopathy [11-14]. Smoking is generally not considered a risk factor; however, at least one study found a significant association between smoking and diabetic macular oedema (DME), a condition that progresses from retinopathy, in people with type 1 diabetes [15]. For decades, the management paradigm for diabetic retinopathy and DME had been early detection, optimal glycaemic control, blood pressure control, laser photocoagulation and surgery, if necessary [3, 16, 17]. More recently, intravitreally administered medications such as antivascular endothelial growth factor (VEGF) agents and corticosteroids have shown beneficial effects [16, 18-20]. However, a number of high-risk participants are not identified by current methods of screening [3].

Lipoprotein-associated phospholipase $\mathrm{A}_{2}\left(\mathrm{Lp}-\mathrm{PLA}_{2}\right)$ is a vascular-specific, proinflammatory enzyme that binds to plasma lipoproteins $(\sim 70-80 \%$ to LDL-cholesterol [LDLc], the rest to HDL-cholesterol). Packard et al first demonstrated, in the West of Scotland Coronary Prevention Study (WOSCOPS) trial, that Lp-PLA 2 activity is associated with increased risk of coronary events [21]. Subsequent studies have confirmed that Lp-PLA 2 activity is prospectively associated with increased risk of coronary heart disease, independent from risk attributable to circulating lipid levels [22, 23].

Lp-PLA ${ }_{2}$ has been postulated to play an important role in diabetes-induced vascular leakage, which may cause the breakdown of the inner blood-retinal barrier that is observed in early and more advanced stages of retinopathy and DME. Studies with Lp-PLA 2 inhibitors, darapladib and SB-435495 (GlaxoSmithKline, King of Prussia, PA, USA), have provided evidence of reduced leakage across the blood-retinal barrier in diabetic participants and a rat model of diabetes, respectively $[24,25]$. In addition, darapladib has been shown to reduce leakage across the blood-brain barrier in diabetic and hypercholesteraemic pig models [26].

The purpose of this study is to explore whether variation in Lp-PLA 2 activity measured in serum samples from a diabetic 
population is associated with subsequent incidence of, or progression from less to more severe, retinopathy.

\section{Methods}

\section{Basic information about the study cohort}

Diabetes Audit and Research in Tayside Scotland (DARTS) is an integrated clinical management system linking all clinical events of individuals with type 2 diabetes in the Tayside region of Scotland and provides complete continuity of care from general physicians, diabetes specialists and population screening services. GoDARTS (Genetics of DARTS) is a subcohort study of individuals from DARTS who have provided a sample of blood for genetic studies and have given consent for linking genetic data to study complications of diabetes, the clinical data for which is continually updated from the electronic medical record [27]. Between 2004 and 2007, participants with type 2 diabetes were recruited (together with nondiabetic controls, who were not included in this study). A serum sample was also collected from every participant at recruitment, and a series of characteristics was measured. Recruitment was treated as the baseline for this study. A cohort study in GoDARTS with a 3 year follow-up period was used to evaluate the primary objective: to test the association between Lp-PLA ${ }_{2}$ activity levels (measured at baseline) and incident diagnosis of retinopathy, as well as progression of retinopathy grade.

\section{Measurement of exposure: Lp-PLA 2 activity}

The exposure of interest was Lp-PLA 2 activity level, measured in serum samples taken at baseline. The biobanked serum samples for this population were analysed for Lp-PLA 2 activity using the CAM colorimetric assay (diaDexus, San Francisco, CA, USA) [28]. Note that Lp-PLA 2 mass was neither measured nor analysed. The activity assay was performed at the Immunoassay Biomarker Core Laboratory, University of Dundee. The diaDexus assay was provided directly from the manufacturer to the laboratory. The intra-assay $\% \mathrm{CV}(n=$ 36 ) was $5.8 \%$. The inter-assay (assay to assay) $\% \mathrm{CV}(n=8)$ was $7.6 \%$. The duplicate intra-assay $\% \mathrm{CV}$ of those participants measured in duplicate $(n=134)$ was $3.0 \%$ (range $0 \%-$ $18.6 \%)$.

\section{Measurement of main outcome: diabetic retinopathy}

For retinopathy, data were acquired from the Scottish National Diabetic Eye Screening Service. The severity of retinopathy was coded as per the Scottish Diabetes Retinal Grading Scheme (www.ndrs-wp.scot.nhs.uk/wp-content/uploads/ 2013/04/Grading-Scheme-2007-v1.1.pdf), and are denoted as follows: disease-free (DR0), mild (DR1), observable (DR2), referable (DR3) and proliferative (DR4). Retinal screening in Tayside, Scotland has been undertaken since 1990, initially with Polaroid images, progressing to digital images in 2000 [10]. The Tayside diabetic retinopathy screening protocol has been described previously [29] and the GoDARTS cohort has been used previously by Liu et al to examine risk factors for the progression of diabetic retinopathy [10]. This cohort provides a high-resolution longitudinal dataset for the study of retinal disease progression. Data includes stages of retinopathy, recorded separately for both eyes, and dates. The eye with more advanced retinopathy stage was used for analysis. The screening data used in this study are from the years 1990 to 2011 . The most recent screening data prior to baseline was used to establish the prevalent retinopathy stage. The primary outcome variables were time to first occurrence in the record of DR1, DR2, DR3 or DR4 (i.e. incident retinopathy). Time to DR2 was assessed as the time from baseline until first visit where grade DR2 was recorded, and similarly for times to DR3 or DR4. However, this study does not examine the association of Lp-PLA $\mathrm{P}_{2}$ with DME, as the screening data does not contain information specific to DME status.

\section{Competing risk: association of Lp-PLA 2 with death in GoDARTS}

Previous studies of diabetic and non-diabetic participants have demonstrated that Lp-PLA ${ }_{2}$ activity levels are strongly associated with incident coronary heart disease and mortality [23]. In GoDARTS, death during the follow-up period was determined from the administrative records of the General Registrar Office, which holds details of deaths throughout Scotland. Time from baseline to death (for the full cohort) was analysed using the Cox proportional hazards model, with age and sex as covariates, and with quartiles of baseline Lp$\mathrm{PLA}_{2}$ activity levels as the exposure (see electronic supplementary material [ESM] Table 1 and ESM Fig. 1).

\section{Exclusion and inclusion criteria}

Exploratory analyses suggested that the modelling assumptions were sensitive to the temporal proximity of death and retinopathy events to the time of serum collection and Lp$\mathrm{PLA}_{2}$ measurement. Therefore, a follow-up period of 3 years was chosen to reduce the potential for bias, while maintaining follow-up for enough incident and progression events to accrue. The main analyses reported considered only outcomes that occurred within a 3 year follow-up period after baseline for each participant. Participants with no diabetic retinopathy assessment, and who also did not die within this follow-up period, were excluded completely from the main analyses. The effect of these exclusions (which made the main analysis 
sample size substantially smaller than the full cohort size) was assessed using sensitivity analyses. Since participants with DR4 at baseline could not be analysed for further disease progression, these participants were also excluded from all analyses. Of the full cohort of $n=6731$ participants, $n=684$ were excluded because they had DR4 at baseline (Table 1), a further $n=4667$ were excluded from the main analyses because they had inadequate follow-up (neither death nor retinopathy assessment within 3 years), and finally 16 individuals were excluded from the analyses as they had inadequate baseline covariate information. Hence 1364 participants were included in the study cohort for analyses.

\section{Statistical methods}

Lp-PLA ${ }_{2}$ measurements were used as a linear trait for association testing with baseline covariates and divided into quartiles for cumulative incidence modelling of survival analysis. Means and SDs of Lp-PLA 2 activity are presented. Diabetic retinopathy grades were the outcome event and were treated as a binary (yes or no) variable for survival analyses. Baseline variables were selected for potential inclusion as covariates, based on known associations with diabetic retinopathy. Variables considered as potential covariates in the analyses were an individual's retinopathy status prior to baseline (estimated from the last visit at or prior to serum collection date), and participant age, sex, prior duration of diabetes, BMI, calculated LDLc, $\mathrm{HbA}_{1 \mathrm{c}}$, smoking status, systolic and diastolic blood pressure (SBP and DBP), eGFR, use of statins and use of diabetes-controlling medication (all measured at baseline). The association with baseline covariates was assessed using univariate linear regression. Variables found to be significantly associated in univariate regressions were then tested in survival analyses, and non-significant variables were eliminated from the final model. Survival analyses adjusted for competing risk of death were conducted with Lp-PLA ${ }_{2}$ activity levels (in quartiles) using a cumulative incidence model. Results of survival analyses are presented as the omnibus test for the Lp$\mathrm{PLA}_{2}$ variable, $\mathrm{HR}$ and $95 \% \mathrm{CI}$. The results of the main effects (unadjusted) and final (adjusted) models on Lp-PLA hazards are shown in Table 2. Individuals with missing data were excluded on a per-analysis basis.

The cumulative incidence graphs presented are unadjusted, and demonstrate the main effect of Lp-PLA 2 quartiles on the

Table 1 Baseline demographics of the full GoDARTS cohort $(n=6731)$ and study cohort $(n=1364)$

\begin{tabular}{|c|c|c|c|c|}
\hline \multirow[t]{2}{*}{ Variable } & \multicolumn{2}{|c|}{ Baseline population $(n=6731)$} & \multicolumn{2}{|c|}{ Study cohort $(n=1364)$} \\
\hline & & $\begin{array}{l}\text { Association with Lp-PLA } 2 \\
\beta \text { estimate }(95 \% \text { CI })\end{array}$ & & $\begin{array}{l}\text { Association with Lp-PLA } 2 \\
\beta \text { estimate }(95 \% \text { CI })\end{array}$ \\
\hline Lp-PLA 2, nmol $\min ^{-1} \mathrm{ml}^{-1}$ & $121.2 \pm 34.8$ & - & $113.4 \pm 32.5$ & - \\
\hline Age, years & $65 \pm 11$ & $-0.41(-0.48,-0.34)$ & $67 \pm 11$ & $-0.38(-0.45,-0.30) * * *$ \\
\hline Sex (\% women) & 44 & $-13.87(-15.51,-12.23) * * *$ & 41 & $-14.00(-15.51,-12.23) * * *$ \\
\hline Smokers ( $\%$ ever smokers) & 62 & $4.33(2.61,6.06)^{*}$ & 63 & $4.33(2.61,6.05)^{* * *}$ \\
\hline Statin users (\% statin users) & 90 & $-9.42(-12.00,-6.85)^{* * *}$ & 91 & $-9.63(-12.60,-6.57) * * *$ \\
\hline Diabetes-controlling medication users ( $\%$ users) & 75 & $-4.58(-6.40,-2.75)$ & 81 & $-4.81(-6.77,-2.86)^{* *}$ \\
\hline Duration of diabetes, years & $7 \pm 6$ & $-0.001(-0.0015,-0.0007)$ & $9 \pm 7$ & $-0.002(-0.0015,-0.0007)^{* * *}$ \\
\hline BMI, $\mathrm{kg} / \mathrm{m}^{2}$ & $31 \pm 6$ & $-0.12(-0.25,-0.98)^{*}$ & $31 \pm 6$ & $-0.16(-0.30,-0.02)^{* * *}$ \\
\hline \multicolumn{5}{|l|}{ Baseline clinical data } \\
\hline Calculated LDLc, mmol/1 & $2.09 \pm 0.77$ & $19.70(18.72,20.67)^{* * *}$ & $2.10 \pm 0.80$ & $20.00(18.54,20.57)^{* * *}$ \\
\hline $\mathrm{HbA}_{1 \mathrm{c}}, \mathrm{mmol} / \mathrm{mol}$ & $58 \pm 16$ & $0.17(0.12,0.22)^{* * *}$ & $59 \pm 18$ & $0.16(0.11,0.22)^{* * *}$ \\
\hline $\mathrm{HbA}_{1 \mathrm{c}}, \%$ & $7.5 \pm 1.45$ & $1.83(1.26,2.40)^{* * *}$ & $7.60 \pm 1.60$ & $1.83(1.26,2.40)^{* * *}$ \\
\hline SBP, mmHg & $142 \pm 19$ & $-0.07(-0.11,-0.30)^{*}$ & $143 \pm 20$ & $-0.08(-0.12,-0.03)$ \\
\hline $\mathrm{DBP}, \mathrm{mmHg}$ & $77 \pm 12$ & $0.28(0.21,0.35)^{* * *}$ & $75 \pm 12$ & $0.27(0.20,0.34)^{* * *}$ \\
\hline 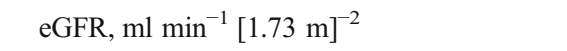 & $85 \pm 20$ & $0.05(0.005,0.099)^{*}$ & $85 \pm 20$ & $0.05(0.006,0.1)^{*}$ \\
\hline \multicolumn{5}{|c|}{ Baseline diabetic retinopathy status (reference group DR0) } \\
\hline $\begin{array}{l}\text { DR0: no diabetic retinopathy, } n(\%) \\
\text { DR1: mild, } n(\%)\end{array}$ & $\begin{array}{l}3207(50.30) \\
1496(23.50)\end{array}$ & \multirow[t]{4}{*}{$-1.44(-2.10,-0.80)^{* * *}$} & $\begin{array}{l}548(40.20) \\
464(34.00)\end{array}$ & \multirow[t]{4}{*}{$-1.44(-2.09,-0.80)^{* * *}$} \\
\hline DR2: moderate, $n(\%)$ & $968(15.20)$ & & $345(25.30)$ & \\
\hline DR3: severe, $n(\%)$ & $16(0.30)$ & & $7(0.50)$ & \\
\hline DR4: proliferative, $n(\%)$ & $684(10.70)$ & & - & \\
\hline
\end{tabular}

Data are mean $\pm \mathrm{SD}, \%$ or $n(\%)$ Associations were tested using univariate linear regression, with Lp-PLA 2 as the outcome

$* * * p<0.001, * * p<0.01, * p<0.05$ 
Table 2 Association of predictors and covariates included in each analysis

\begin{tabular}{|c|c|c|c|}
\hline Variable & HR & $95 \% \mathrm{CI}$ & $p$ value \\
\hline \multicolumn{4}{|c|}{ Development of incident retinopathy from disease-free state ${ }^{a}$} \\
\hline \multicolumn{4}{|c|}{ Main effects model } \\
\hline Lp-PLA 2 Q2 vs Q1 & 1.33 & $1.08,1.64$ & $<0.01$ \\
\hline Lp-PLA 2 Q3 vs Q1 & 1.56 & $1.28,1.90$ & $<0.0001$ \\
\hline Lp-PLA 2 Q4 vs Q1 & 1.52 & $1.24,1.86$ & $<0.0001$ \\
\hline \multicolumn{4}{|l|}{ Final model $\mathrm{l}^{\mathrm{b}}$} \\
\hline Lp-PLA 2 Q2 vs Q1 & 1.52 & $1.21,1.91$ & $<0.001$ \\
\hline Lp-PLA 2 Q3 vs Q1 & 1.72 & $1.37,2.17$ & $<0.001$ \\
\hline Lp-PLA 2 Q4 vs Q1 & 2.08 & $1.64,2.63$ & $<0.001$ \\
\hline \multicolumn{4}{|c|}{ Progression to observable or more severe retinopathy from lower grade } \\
\hline \multicolumn{4}{|l|}{ Main effects model } \\
\hline Lp-PLA 2 Q2 vs Q1 & 1.86 & $1.29,2.77$ & $<0.01$ \\
\hline Lp-PLA 2 Q3 vs Q1 & 2.33 & $1.59,3.42$ & $<0.0001$ \\
\hline Lp-PLA 2 Q4 vs Q1 & 2.21 & $1.46,3.331$ & $<0.001$ \\
\hline \multicolumn{4}{|l|}{ Final model ${ }^{\mathrm{d}}$} \\
\hline Lp-PLA 2 Q2 vs Q1 & 1.96 & $1.23,3.00$ & $<0.01$ \\
\hline Lp-PLA 2 Q3 vs Q1 & 2.71 & $1.75,4.20$ & $<0.001$ \\
\hline Lp-PLA 2 Q4 vs Q1 & 2.82 & $1.71,4.65$ & $<0.001$ \\
\hline \multicolumn{4}{|c|}{ Progression to referable or proliferative retinopathy from lower grades } \\
\hline \multicolumn{4}{|l|}{ Main effects model } \\
\hline Lp-PLA 2 Q2 vs Q1 & 1.76 & $1.23,2.50$ & $<0.01$ \\
\hline Lp-PLA 2 Q3 vs Q1 & 1.81 & $1.26,2.60$ & $<0.01$ \\
\hline Lp-PLA 2 Q4 vs Q1 & 1.83 & $1.25,2.70$ & $<0.01$ \\
\hline \multicolumn{4}{|l|}{ Final model ${ }^{\mathrm{f}}$} \\
\hline Lp-PLA 2 Q2 vs Q1 & 1.64 & $1.13,2.37$ & $<0.01$ \\
\hline Lp-PLA 2 Q3 vs Q1 & 1.98 & $1.34,2.92$ & $<0.01$ \\
\hline Lp-PLA 2 Q4 vs Q1 & 1.87 & $1.26,2.77$ & $<0.01$ \\
\hline
\end{tabular}

${ }^{\mathrm{a}} \mathrm{DR} 0$ to DR1 or higher, $n=1013$

${ }^{\mathrm{b}} \mathrm{Lp}_{\mathrm{PLA}}$ omnibus test for variable: Wald $\chi^{2}, 38.2(\mathrm{df}=3) p$ value $=3 \times$ $10^{-8}$

${ }^{\mathrm{c}}$ DR0 or DR1 to DR2 or higher, $n=1241$

${ }^{\mathrm{d}}$ Lp-PLA 2 omnibus test for variable: Wald $\chi^{2}, 23.6(\mathrm{df}=3) p$ value $=$ $1.5 \times 10^{-53}$

${ }^{\mathrm{e}} \mathrm{DR} 0, \mathrm{DR} 1$ or DR2 to DR3 or higher, $n=1364$

${ }^{\mathrm{f}} \mathrm{Lp}-\mathrm{PLA}_{2}$ omnibus test for variable: Wald $\chi^{2}, 16.8(\mathrm{df}=3) p$ value $=$ $2.8 \times 10^{-3}$

$\mathrm{Q}$, quartile

development and progression of retinopathy. All HRs of Lp$\mathrm{PLA}_{2}$ use the lowest quartile of Lp-PLA ${ }_{2}$ activity as the reference. All statistical analyses were conducted using SAS 9.4 (SAS institute, Cary, NC, USA).

\section{Ethics approval and participant consent}

The Tayside Medical Ethics Committee has approved the GoDARTS study and informed consent was obtained for all participants. The participants have consented to research on their samples and data related to diabetes, its treatment and related conditions. They have also consented to the fact that the studies may involve collaborative studies with commercial companies and that the participants will not benefit financially from such collaborations. Neither the University of Dundee research team nor GlaxoSmithKline had access to personally identifiable information. All event level data is provided to the University of Dundee research team in anonymised fashion by the Data Linkage team of the Health Informatics Centre at the University of Dundee.

\section{Results}

\section{Baseline associations}

All diabetic participants in GoDARTS with available serum were assayed for Lp-PLA ${ }_{2}$ activity. Measured Lp-PLA ${ }_{2}$ activity levels were approximately normally distributed (presented in Fig. 1a) and were therefore analysed as an untransformed, continuous variable for baseline analysis. The mean (SD) Lp$\mathrm{PLA}_{2}$ activity level was 121 (35) nmol min ${ }^{-1} \mathrm{ml}^{-1}$. The observed quartiles were $\mathrm{Q} 1 \leq 97.2, \mathrm{Q} 2 \leq 117.3, \mathrm{Q} 3 \leq 140.7$ and $\mathrm{Q} 4 \leq 377.9 \mathrm{nmol} \mathrm{min}{ }^{-1} \mathrm{ml}^{-1}$, which were used to divide the participants into equal sized groups for survival analyses.

Their sex, age, usage of diabetes-controlling medication, usage of statins, smoking status, duration of diabetes, $\mathrm{HbA}_{1 \mathrm{c}}$, LDLc levels, eGFR and SBP were all associated with their Lp-PLA ${ }_{2}$ activity levels. The distribution of Lp$\mathrm{PLA}_{2}$, and the association of sex, BMI, LDLc and $\mathrm{HbA}_{1 \mathrm{c}}$ with Lp-PLA 2 in the population is presented in Fig. 1. Lp$\mathrm{PLA}_{2}$ levels stratified by sex showed significantly different levels; women have lower levels than men (Fig. 1b). The mean (SD) Lp-PLA 2 levels amongst women was 114 (35) nmol min ${ }^{-1} \mathrm{ml}^{-1}$ compared with 127 (35) nmol min ${ }^{-1} \mathrm{ml}^{-1}$ for men. The correlation of Lp-PLA 2 activity levels with BMI, LDLc, $\mathrm{HbA}_{1 \mathrm{c}}$ and eGFR at baseline are also shown in Fig. 1c-f, respectively. The only notable correlation was with LDLc, which showed a strong linear relationship with Lp-PLA 2 , with a correlation coefficient of 0.44 .

The association of these potentially confounding variables with Lp-PLA ${ }_{2}$ is presented for the study population and the full GoDARTS cohort (Table 1). Variables showed similar associations in the study population compared with the full GoDARTS cohort; however, the study cohort were on average 2 years older, had type 2 diabetes for 2 years longer, a higher proportion were medicated for type 2 diabetes, and a lower proportion were disease-free (DR0) at baseline. This suggests that the study cohort had more severe disease at baseline and were therefore more likely to be screened regularly for progression during follow-up. 
Fig. 1 (a) Baseline distribution of Lp-PLA ${ }_{2}$ activity

$\left(\mathrm{nmol} \mathrm{min}{ }^{-1} \mathrm{ml}^{-1}\right)$. (b)

Distribution by sex $(\beta=-0.12$, $p<0.0001)$. (c) Correlation with BMI $\left(\mathrm{kg} / \mathrm{m}^{2}\right)(r=-0.02, p=$ 0.07). (d) Correlation with LDLc $(\mathrm{mmol} / \mathrm{l})(r=0.44, p<0.0001)$. (e) Correlation with $\mathrm{HbA}_{1 \mathrm{c}}$ $(\mathrm{mmol} / \mathrm{mol})(r=0.08, p=$ 0.0003). (f) Correlation with eGFR $\left(\mathrm{ml} \mathrm{min}^{-1}[1.73 \mathrm{~m}]^{-2}\right)(r=$ $0.03, p=0.026)$. F, female; $\mathrm{M}$, male a

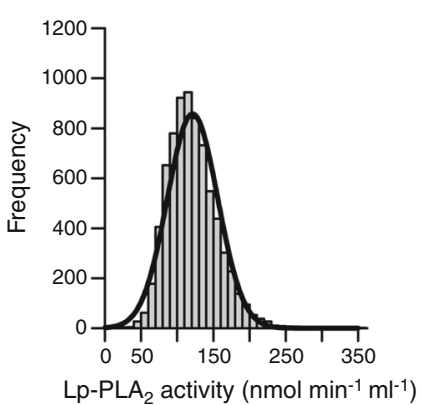

d

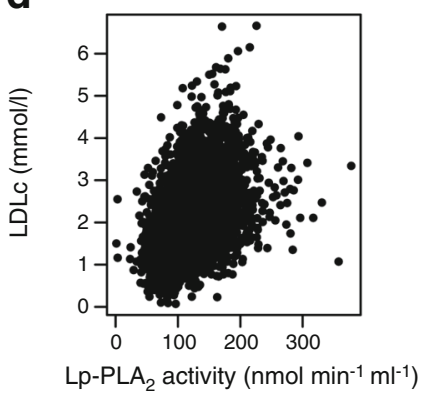

b

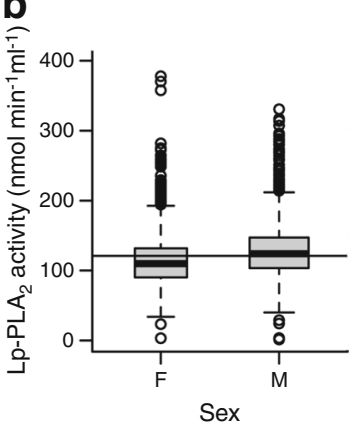

e

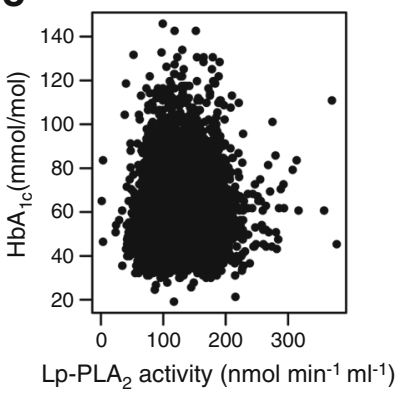

C

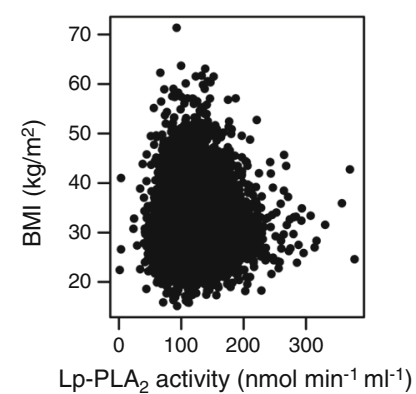

f

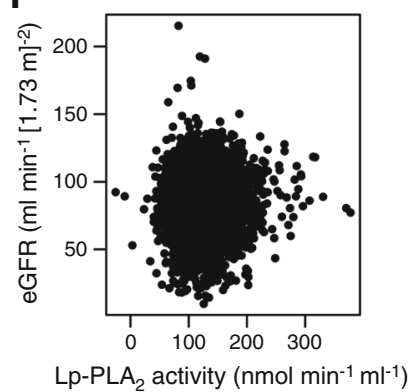

\section{Association of Lp-PLA 2 with death}

In agreement with results from previous large meta-analyses including both diabetic and non-diabetic participants [23], Lp$\mathrm{PLA}_{2}$ activity levels were strongly associated with death in the diabetic population of the GoDARTS cohort for whom Lp$\mathrm{PLA}_{2}$ activity was measured $(n=6731)$. This is demonstrated in ESM Fig. 1 and ESM Table 1 as an increased risk of death for participants in the highest two quartiles of Lp-PLA 2 levels compared with the lowest. The hazard of death for those in the highest Lp-PLA 2 quartile was approximately one and a half times that for participants in the lowest quartile (HR 1.45, 95\% CI $1.24,1.68 ; p<0.001)$. Hence, when analysing the association between Lp-PLA 2 activity levels and time to progression, censoring at death would violate the non-informative censoring assumption required for standard survival analyses such as Cox regression [30]. Therefore, a competing risk survival analysis [31] was used to analyse the association with incident diabetic retinopathy and progression to more severe stages.

\section{Survival analyses}

\section{Association of Lp-PLA 2 activity with incident diabetic retinop-} athy For this analysis, a cohort of 1013 individuals who had no observable retinopathy (DR0) at baseline were included. Of these, 676 individuals progressed to any retinopathy grade (mild retinopathy: DR1, or higher) in the 3 year follow-up period. In the same cohort there were 143 deaths prior to any record of progression, and 194 individuals who were censored (alive and without progression).

As shown in Table 2, there was a progressive trend of increased risk across the quartiles. The hazards of developing incident retinopathy were $1.52,1.72$ and 2.08 for $\mathrm{Lp}_{\mathrm{PLA}}$ quartiles 2, 3 and 4, respectively, compared with quartile 1. The omnibus test of hazard across the quartiles of Lp-PLA activity was highly significant $\left(p=3 \times 10^{-8}\right)$. This analysis was adjusted for sex, diabetes-controlling medication, use of statins, $\mathrm{HbA}_{1 \mathrm{c}}$ levels, systolic blood pressure, LDLc levels and the age of the individual at baseline and was therefore independent of traditional risk factors for diabetic retinopathy.

The accompanying cumulative incidence plot (Fig. 2a) is unadjusted and shows the increased hazards of incident retinopathy for Lp-PLA 2 activity quartiles relative to the lowest quartile (light blue line).

The GoDARTS study largely recruited at the time of the participants' annual diabetes review, which accounts for the evident increase in retinopathy diagnosis at the 1 year mark, coinciding with the next annual review after recruitment.

Association of Lp-PLA 2 activity with progression to observable or more severe diabetic retinopathy from lower grades For this analysis, a cohort of 1241 individuals who had no observable retinopathy or had mild retinopathy (DR0 or DR1) at baseline were included. Of these, 209 individuals progressed to more severe retinopathy (grade DR2 or higher) 

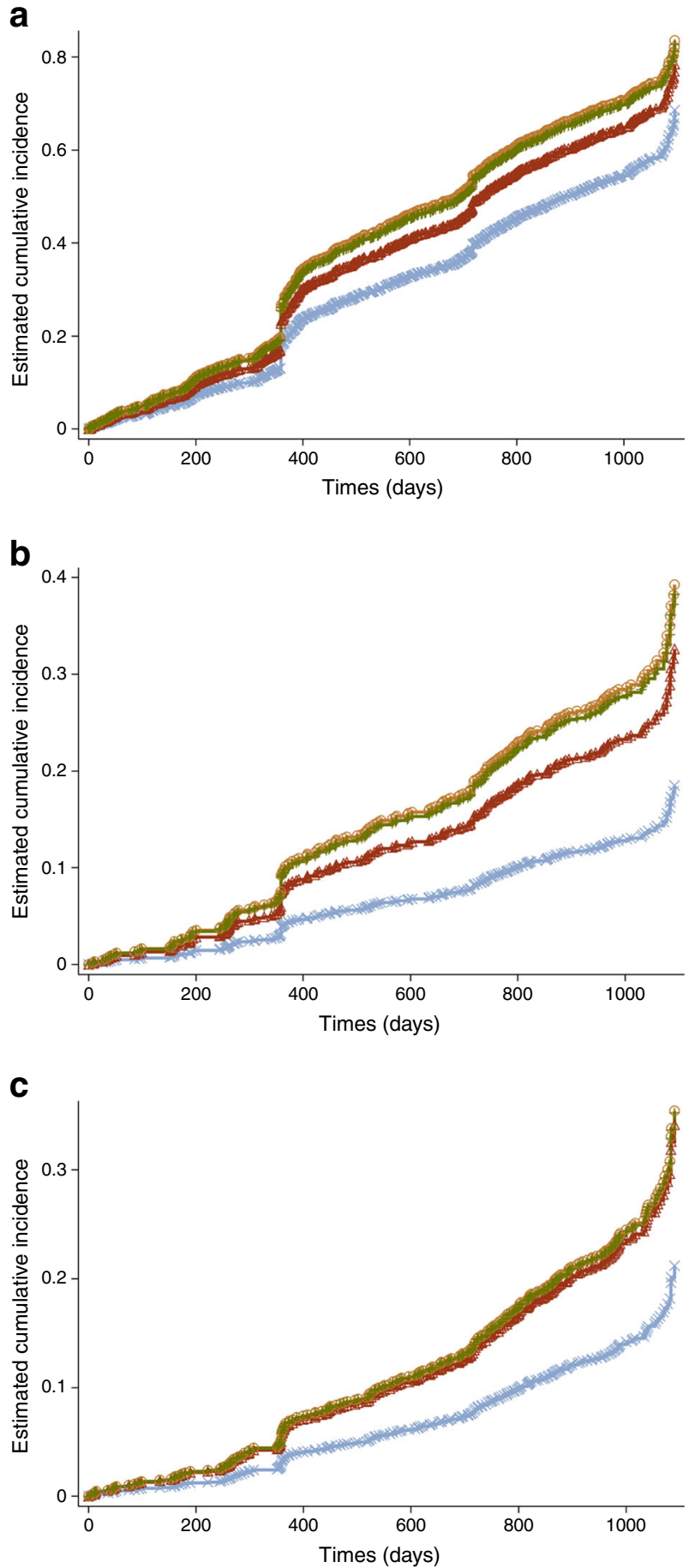

Fig. 2 (a) Cumulative incidence plot of the hazards of incident diabetic retinopathy for Lp-PLA 2 activity quartiles, main effects model. (b) Cumulative incidence plot of the hazards of progression to observable or more severe retinopathy by Lp-PLA 2 activity quartiles, main effects model. (c) Cumulative incidence plot of hazards of progression to referable or proliferative retinopathy by Lp-PLA 2 activity quartiles, main effects model. Light blue line with crosses, lowest quartile (quartile 1) of Lp-PLA $A_{2}$ activity; red line with triangles, quartile 2; brown line with circles, quartile 3; green line with crosses, highest quartile (quartile 4). Symbols denote events in the 3 year follow-up period. In the same cohort there were 432 participants who died prior to progression, and 600 individuals who were censored.

The hazards of developing moderate or more severe retinopathy were $1.96,2.71$ and 2.82 for Lp-PLA 2 activity quartiles 2, 3 and 4, respectively, compared with quartile 1 (Table 2). The overall omnibus test for association across the quartiles of Lp-PLA 2 activity was highly significant ( $p=$ $1.5 \times 10^{-5}$ ) and independent of traditional risk factors for diabetic retinopathy. This analysis was adjusted for diabetescontrolling medication, $\mathrm{HbA}_{1 \mathrm{c}}$ levels, SBP, LDLc levels and the age of the individual and was therefore independent of traditional risk factors.

The accompanying cumulative incidence plot (Fig. 2b) is unadjusted and shows the increased hazards of developing moderate or more severe forms of retinopathy by Lp-PLA 2 quartiles compared with the lowest quartile (light blue line).

Association of Lp-PLA 2 activity with progression to referable or proliferative diabetic retinopathy from lower grades For this analysis, the cohort of 1364 individuals who had no observable retinopathy, or had mild or observable retinopathy (DR0, DR1, or DR2) at baseline were included. Of these, 461 individuals progressed to more severe retinopathy (grade DR3 or higher) in the 3 year follow-up period. In the same cohort there were 435 participants who died prior to progression, and 468 individuals who were censored.

The hazards of developing severe retinopathy were 1.64 , 1.98 and 1.87 for Lp-PLA 2 quartiles 2, 3 and 4 respectively compared with quartile 1 (Table 2). The omnibus test of association across the quartiles of Lp-PLA ${ }_{2}$ activity levels was highly significant $\left(p=2.8 \times 10^{-3}\right)$ and independent of traditional risk factors for diabetic retinopathy. This analysis was adjusted for grade at baseline, diabetes-controlling medication, $\mathrm{HbA}_{1 \mathrm{c}}$ levels, SBP, smoking status and the age of the individual and therefore independent of traditional risk factors.

The cumulative incidence plot (Fig. 2c) is unadjusted and shows the increased hazards of progressing to referable or proliferative retinopathy from lower grades by Lp-PLA 2 quartiles compared with the lowest quartile (light blue line).

ESM Table 2 shows HRs for Lp-PLA 2 quartiles for each analysis with every level of adjustment.

\section{Sensitivity analyses for extended follow-up period}

When considering the longer follow-up period of 5 years, the association with Lp-PLA $\mathrm{P}_{2}$ activity with incident diabetic retinopathy was attenuated (HR 1.17, 1.14 and 1.26 for Lp-PLA 2 quartiles 2, 3 and 4 respectively) with only the highest quartile remaining significant $(p=0.03)$. Further details are provided in ESM Table 3 and ESM Fig. 2. Therefore, it was decided to limit our analysis to a 3 year follow-up period. 


\section{Discussion}

As a result of the association of Lp-PLA $\mathrm{PL}_{2}$ with increased risk for cardiovascular disease [23] we observe that high Lp-PLA 2 activity levels are prospectively associated with an increased risk of death in this cohort of individuals with type 2 diabetes. All analyses for retinopathy outcomes were therefore adjusted for this factor by using a competing risk analysis, which directly estimates the effect of Lp-PLA 2 activity separately on the event of interest (incidence or progression) and the competing event (death), and also allows non-informative censoring for participants when neither event occurred before the end of the study period.

Lp-PLA $A_{2}$ activity was an independent predictor for development of incident diabetic retinopathy. Analysing progression to any grade resulted in a similar finding. The association of baseline Lp-PLA $\mathrm{P}_{2}$ activity with incidence and progression of diabetic retinopathy was highly statistically significant and was independent of LDLc and other traditional risk factors including $\mathrm{HbA}_{1 \mathrm{c}}$, blood pressure, lipid-controlling medication, diabetes-controlling medication, age and sex. A progressive trend of risk by Lp-PLA $\mathrm{P}_{2}$ activity quartiles can be observed in the first two analyses (incident diabetic retinopathy and progression to observable or more severe grades), where every subsequent quartile confers additional risk to the development of retinopathy or the progression to more severe stages.

Previously, well-established risk factors for diabetic retinopathy included duration of diabetes and poor glycaemic control and blood pressure. This study presents evidence that a proinflammatory enzyme is associated with both the incidence of diabetic retinopathy, as well as progression to more severe stages. Crucially, this is a potential therapeutic target, for which existing antagonists could be repurposed.

The conclusions of these results are supported by the Bradford Hill causal criteria for epidemiological studies [32]: the associations are robust (statistically significant and do not change drastically with the addition of covariates), consistent (across all analyses, albeit not externally validated) and show specificity to Lp-PLA 2 in models adjusted for potential confounders. Additionally, the results are temporally valid since the measurement of exposure $\left(\mathrm{Lp}-\mathrm{PLA}_{2}\right)$ precedes the outcome (retinopathy) and the association is sensitive to the time between the two, with attenuation on increasing time from the date of the activity measure (it is easier to predict the immediate future). The association shows a biological gradient (higher quartiles of Lp-PLA $A_{2}$ levels are associated with increased hazards of incidence and progression). There is a plausible mechanism for the association as being due to vascular leakage, and experimental evidence (from animal studies) supports the hypothesis that inhibition of Lp-PLA results in reduced leakage across the blood-retinal and blood-brain barriers [24-26]. The findings of this study will have to be replicated externally in other cohorts.

Limitations of this study include the fact that serum was collected at a random point in the disease progression for each individual, so it was not consistent by disease state. In addition, $10 \%$ of the cohort had proliferative retinopathy at the time of serum collection, hence data from these participants were uninformative in a prospective analysis of incidence. A further $70 \%$ of the full cohort had inadequate follow-up for the main analyses, with neither death nor retinopathy assessment in 3 years after baseline. However, this would be an interesting area for exploration, given the evidence presented here. Sensitivity analyses using longer follow-up periods benefited from larger sample size (fewer participants with inadequate follow-up) but potentially introduced other biases, such as increased competing risks and a longer gap between measurement of Lp-PLA ${ }_{2}$ and occurrence of outcome. It is possible that the association between Lp-PLA ${ }_{2}$ activity and onset or progression of diabetic retinopathy is confounded (by factors unknown or unmeasured at baseline) or is biased (e.g. by another unidentified competing risk, or by another mechanism of informative censoring). The nature of an observational epidemiological study means that it is impossible to rule out the possibility of such confounding or biases.

However, complete records of retinopathy screening data for all individuals in a large cohort of individuals with diabetes is a powerful resource. A further strength is the rich set of clinical and questionnaire baseline data that can be used to adjust for known or suspected confounders, and outcome data that can be used to adjust for competing risks.

\section{Interpretation}

Lp-PLA 2 activity levels are shown to be associated with an individual's risk of developing diabetic retinopathy. Individuals with Lp-PLA ${ }_{2}$ activity levels above the median have a very high risk of progressing to more severe stages. Since this relationship appears to be independent of traditional risk factors and, specifically, independent of baseline LDLc levels, these data are consistent with the hypothesis that systemic inhibition of Lp-PLA $\mathrm{P}_{2}$ activity may be a good therapeutic target in the prevention of this complication of diabetes.

\section{Application}

This is a population cohort of white Scottish individuals, with many variables captured using routinely collected health record information. The study population is representative of the diabetic population in Scotland undergoing routine screening [10]. The duration of type 2 diabetes is an important risk factor when considering the 
extrapolation of these results; diabetic retinopathy is more prevalent when duration of type 2 diabetes is longer than 10 years [2]. This is reflected in the duration of type 2 diabetes in the study population, which, with the addition of 3 years of follow-up, is on average 12 years. Furthermore, the rate of adherence to annual, recommended screens for those with pre-existing retinopathy was reported to be as low as $61 \%$, while the rate of screening for those with long duration of type 2 diabetes was $57 \%$ [33]. Overall there is evidence to suggest that adherence to annual screens range between $34 \%$ and $65 \%$ [3, 9, 33-35]. This suggests that the drop-off rate noted with the 3 year follow-up exclusion criteria is reflective of real-world consumption of healthcare in a population with type 2 diabetes. Further, association characteristics of Lp$\mathrm{PLA}_{2}$ activity are similar to those reported elsewhere [21-23]. Therefore the generalisability of the study to a population of European descent with routine access to healthcare is likely to be high. On the basis of previous observations in animal and human studies it is unlikely that the ethnicity of the cohort would limit the generalisability of this study.

Acknowledgements We are grateful to all the participants in this study, the GoDARTS team for their help in recruiting the participants, and to the whole team, including interviewers, computer and laboratory technicians, clerical workers, research scientists, volunteers, managers, receptionists and nurses. The study complies with the Declaration of Helsinki. We acknowledge the support of the Health Informatics Centre, University of Dundee for managing and supplying the anonymised data and NHS Tayside, the original data owner.

Data availability Data is available through application to the GoDARTS data acquisition committee.

Funding This study was funded by GlaxoSmithKline. The GoDARTS study was funded by The Wellcome Trust (Award 072960 and 084726) and the UK Medical Research Council (Award G0601261). The funders were involved in study design and provided editorial assistance.

Duality of interest TJ, MMM and REW are employees of GlaxoSmithKline. CNAP received a research grant from GlaxoSmithKline and MKS was paid through same grant. The other authors declare that there is no duality of interest associated with their contribution to this manuscript.

Contribution statement MKS and CNAP made substantial contributions to conception and design, acquisition of data, analysis and interpretation of data, drafting the article and revising it critically for important intellectual content. GK and FC made substantial contributions to acquisition of data, analysis and interpretation of data, and revising the article critically for important intellectual content. ASFD made substantial contributions to conception and design, analysis and interpretation of data, and revising the article critically for important intellectual content. EP and ADM made substantial contributions to conception and design and acquisition of data, and revising the article critically for important intellectual content. TJ, MMM and REW made substantial contributions to conception and design, analysis and interpretation of data, and revising the manuscript critically for important intellectual content. All authors gave final approval of the version to be published. CNAP is the guarantor of this work.
Open Access This article is distributed under the terms of the Creative Commons Attribution 4.0 International License (http:// creativecommons.org/licenses/by/4.0/), which permits unrestricted use, distribution, and reproduction in any medium, provided you give appropriate credit to the original author(s) and the source, provide a link to the Creative Commons license, and indicate if changes were made.

\section{References}

1. Williams R, Airey M, Baxter H et al (2004) Epidemiology of diabetic retinopathy and macular oedema: a systematic review. Eye (Lond) 18:963-983

2. Yau JWY, Rogers SL, Kawasaki R et al (2012) Global prevalence and major risk factors of diabetic retinopathy. Diabetes Care 35: 556-564

3. Ciulla TA, Amador AG, Zinman B (2003) Diabetic retinopathy and diabetic macular edema: pathophysiology, screening, and novel therapies. Diabetes Care 26:2653-2664

4. Chen E, Looman M, Laouri M et al (2010) Burden of illness of diabetic macular edema: literature review. Curr Med Res Opin 26: $1587-1597$

5. Lopes de Faria JM, Jalkh AE, Trempe CL, McMeel JW (1999) Diabetic macular edema: risk factors and concomitants. Acta Ophthalmol Scand 77:170-175

6. Varma R (2016) Diabetic retinopathy: challenges and future directions. Am J Ophthalmol 141:539-541

7. Miljanovic B, Glynn RJ, Nathan DM, Manson JE, Schaumberg DA (2004) A prospective study of serum lipids and risk of diabetic macular edema in type 1 diabetes. Diabetes 53:2883-2892

8. Zheng Y, Lamoureux EL, Lavanya R et al (2012) Prevalence and risk factors of diabetic retinopathy in migrant Indians in an urbanized society in Asia: the Singapore Indian eye study. Ophthalmology 119:2119-2124

9. Klein R, Knudtson MD, Lee KE, Gangnon R, Klein BE (2009) The Wisconsin Epidemiologic Study of Diabetic Retinopathy XXIII: the twenty-five-year incidence of macular edema in persons with type 1 diabetes. Ophthalmology 116:497-503

10. Liu Y, Wang M, Morris AD et al (2013) Glycemic exposure and blood pressure influencing progression and remission of diabetic retinopathy: a longitudinal cohort study in GoDARTS. Diabetes Care 36:3979-3984

11. Klein R, Klein BE, Moss SE, Davis MD, DeMets DL (1988) Glycosylated hemoglobin predicts the incidence and progression of diabetic retinopathy. JAMA 260:2864-2871

12. Vitale S, Maguire MG, Murphy RP et al (1995) Clinically significant macular edema in type I diabetes. Incidence and risk factors. Ophthalmology 102:1170-1176

13. Klein R, Klein BE, Moss SE, Cruickshanks KJ (1998) The Wisconsin Epidemiologic Study of Diabetic Retinopathy: XVII. The 14-year incidence and progression of diabetic retinopathy and associated risk factors in type 1 diabetes. Ophthalmology 105:1801-1815

14. Chew EY, Klein ML, Ferris FL 3rd et al (1996) Association of elevated serum lipid levels with retinal hard exudate in diabetic retinopathy: Early Treatment Diabetic Retinopathy Study (ETDRS) report 22. Arch Ophthalmol 114:1079-1084

15. Kramer CK, de Azevedo MJ, da Costa Rodrigues T, Canani LH, Esteves J (2008) Smoking habit is associated with diabetic macular edema in type 1 diabetes mellitus patients. J Diabetes Complicat 22: 430

16. The Diabetic Retinopathy Clinical Research Network, Elman MJ, Aiello LP et al (2010) Randomized trial evaluating ranibizumab 
plus prompt or deferred laser or triamcinolone plus prompt laser for diabetic macular edema. Ophthalmology 117:1064-1077

17. Furlani BA, Meyer CH, Rodrigues EB et al (2007) Emerging pharmacotherapies for diabetic macular edema. Expert Opin Emerg Drugs 12:591-603

18. Johnson MW (2009) Etiology and treatment of macular edema. Am J Ophthalmol 147:11-21

19. Mitchell P, Bandello F, Schmidt-Erfurth U et al (2011) The RESTORE study: ranibizumab monotherapy or combined with laser versus laser monotherapy for diabetic macular edema. Ophthalmology 118:615-625

20. Nguyen QD, Brown DM, Marcus DM et al (2012) Ranibizumab for diabetic macular edema: results from 2 phase III randomized trials: RISE and RIDE. Ophthalmology 119:789-801

21. Packard CJ, O'Reilly DS, Caslake MJ et al (2000) Lipoproteinassociated phospholipase $\mathrm{A} 2$ as an independent predictor of coronary heart disease. West of Scotland Coronary Prevention Study Group. N Engl J Med 343:1148-1155

22. Heart Protection Study Collaborative Group (2010) Lipoproteinassociated phospholipase A2 activity and mass in relation to vascular disease and nonvascular mortality. J Intern Med 268:348-358

23. The Lp-PLA2 Studies Collaboration, Thompson A, Gao P et al (2010) Lipoprotein-associated phospholipase A2 and risk of coronary disease, stroke, and mortality: collaborative analysis of 32 prospective studies. Lancet 375:1536-1544

24. Staurenghi G, Ye L, Magee MH et al (2015) Darapladib, a lipoprotein-associated phospholipase A2 inhibitor, in diabetic macular edema: a 3-month placebo-controlled study. Ophthalmology 122:990-996

25. Canning P, Kenny B-A, Prise V et al (2016) Lipoprotein-associated phospholipase A2 (Lp-PLA $)$ as a therapeutic target to prevent retinal vasopermeability during diabetes. Proc Natl Acad Sci U S A $113: 7213-7218$
26. Acharya NK, Levin EC, Clifford PM et al (2013) Diabetes and hypercholesterolemia increase blood-brain barrier permeability and brain amyloid deposition: beneficial effects of the LpPLA2 inhibitor darapladib. J Alzheimers Dis 35:179-198

27. Hébert HL, Shepherd B, Milburn K et al (2017) Cohort profile: Genetics of Diabetes Audit and Research in Tayside Scotland (GoDARTS). Int J Epidemiol https://doi.org/10.1093/ije/dyx140

28. Koenig W, Twardella D, Brenner H, Rothenbacher D (2006) Lipoprotein-associated phospholipase A2 predicts future cardiovascular events in patients with coronary heart disease independently of traditional risk factors, markers of inflammation, renal function, and hemodynamic stress. Arterioscler Thromb Vasc Biol 26: $1586-1593$

29. Leese GP, Morris AD, Swaminathan K et al (2005) Implementation of national diabetes retinal screening programme is associated with a lower proportion of patients referred to ophthalmology. Diabet Med 22:1112-1115

30. Cox DR (1972) Regression models and life-tables. J R Stat Soc Ser B 34:187-220

31. Fine JP, Gray RJ (1999) A proportional hazards model for the subdistribution of a competing risk. J Am Stat Assoc 94:496-509

32. Hill AB (1965) The environment and disease: association or causation? Proc R Soc Med 58:295-300

33. Brechner RJ, Cowie CC, Howie LJ et al (1993) Ophthalmic examination among adults with diagnosed diabetes mellitus. JAMA 270: 1714-1718

34. Mukamel DB, Bresnick GH, Wang Q, Dickey CF (1999) Barriers to compliance with screening guidelines for diabetic retinopathy. Ophthalmic Epidemiol 6:61-72

35. Schoenfeld ER, Greene JM, Wu SY, Leske MC (2001) Patterns of adherence to diabetes vision care guidelines: baseline findings from the Diabetic Retinopathy Awareness Program. Ophthalmology 108: $563-571$ 